\title{
Whole genome expression profiling and screening for differentially expressed cytokine genes in human bone marrow endothelial cells treated with humoral inhibitors in liver cirrhosis
}

\author{
BO GAO $^{1}$, WANG SUN ${ }^{1}$, XIANQI WANG ${ }^{2}, \mathrm{XU} \mathrm{JIA}^{3}$, BIAO MA $^{1}$, \\ YU CHANG ${ }^{1}$, WEIHUI ZHANG ${ }^{1}$ and DONGBO XUE ${ }^{1}$ \\ ${ }^{1}$ Department of General Surgery, The First Affiliated Hospital of Harbin Medical University;
${ }^{2}$ Department of Gastroenterology, The Second Affiliated Hospital of Harbin Medical University;
${ }^{3}$ College of Bioinformatics Science and Technology of Harbin Medical University, Harbin, P.R. China
}

Received June 6, 2013; Accepted September 6, 2013

DOI: 10.3892/ijmm.2013.1495

\begin{abstract}
Bone marrow endothelial cells (BMECs) are important components of the hematopoietic microenvironment in bone marrow, and they can secrete several types of cytokines to regulate the functions of hematopoietic stem/progenitor cells. To date, it is unknown whether BMECs undergo functional changes and lead to hematopoietic abnormalities in cases of liver cirrhosis (LC). In the present study, whole genome microarray analysis was carried out to detect differentially expressed genes in human BMECs treated for $48 \mathrm{~h}$ with medium supplemented with $20 \%$ pooled sera from 26 patients with LC or 10 healthy volunteers as the control group. A total of 1,106 upregulated genes and 766 downregulated genes were identified. In Gene Ontology analysis, the most significant categories of genes were revealed. A large number of the upregulated genes were involved in processes, such as cell-cell adhesion, apoptosis and cellular response to stimuli and the downregulated genes were involved in the negative regulation of secretion, angiogenesis, blood vessel development and cell growth. Pathway analysis revealed that the upregulated genes were either cell adhesion molecules or parts of the apoptotic signaling pathway and the downregulated genes were involved in the Wnt signaling pathway and MAPK signaling pathway. These were the pathways with the highest enrichment scores. The results of apoptosis assays revealed that the humoral inhibitors in the sera of patients with LC induced the apoptosis of BMECs, which confirmed the accuracy of bioinformatic analysis. Moreover, we screened and verified 21 differentially expressed cytokine
\end{abstract}

Correspondence to: Professor Weihui Zhang or Professor Dongbo Xue, Department of General Surgery, The First Affiliated Hospital of Harbin Medical University, 23 You Zheng Street, Harbin 150001, P.R. China

E-mail: zhangweihui626@hotmail.com

E-mail: xue9971@sina.com

Key words: liver cirrhosis, bone marrow endothelial cells, whole genome microarray, cy tokine, hematological abnormalities, apoptosis genes [transforming growth factor (TGF)B1, tumor necrosis factor (TNF)B, TNF receptor superfamily, member $11 \mathrm{~b}$ (TNFRSF11B), TNF (ligand) superfamily, member 13b (TNFSF13B), interleukin (IL)1A, IL6, IL11, IL17C, IL24, family with sequence similarity 3 , member B (FAM3B), Fas ligand (FASLG), matrix metallopeptidase (MMP)3, MMP15, vitronectin (VTN), insulin-like growth factor 1 (IGF1), fibroblast growth factor 22 (FGF22), slit homolog 2 (Drosophila) (SLIT2), thrombospondin (THBS)2, THBS3, chemokine (C-C motif) ligand 28 (CCL28) and macrophage stimulating 1 (MST1)] from 97 cytokine genes in BMECs treated with serum from patients with LC. The results from our study demonstrate that the humoral inhibitors in the sera of patients with LC induce the dysfunction and abnormal cytokine secretion by BMECs, which may be a novel mechanism responsible for hematological abnormalities in patients with LC.

\section{Introduction}

Hematological abnormalities are frequently observed in patients with liver cirrhosis (LC). It has been reported that $84 \%$ of patients with the same type of LC and graded as Child-Pugh A/B for liver function suffer from hematological abnormalities, among which $32 \%$ of patients suffer from decreases in several types of blood cells, and the remainder are mainly patients with thrombocytopenia or combined thrombocytopenia and granulocytopenia (1). Moreover, these may lead to serious complications and may thus affect the prognosis of patients. They may be the limiting factors in corresponding invasive surgeries, such as liver puncturation, liver biopsy, endoscopy or surgical treatments. Leucopenia may increase the risk of infection following surgery. Thrombocytopenia may increase the risk of esophageal variceal hemorrhage and hemorrhage during and after surgery, while anemia may lead to even more complications after hemorrhagic events (2). Some studies have reported the incidence of these abnormal situations, such as hypersplenia and a decrease in thrombopoietin in the liver, which further leads to aleukia (3). Portal hypertension causes alimentary tract hemorrhaging, hemolysis and the loss of hematopoietic substances, such as ferrum, folic acid and other substances, which further leads to 
anemia of organisms (4). Moreover, hepatitis B and C viruses, excessive alcohol abuse and drug intake induce the formation of LC and subsequently impair hematopoietic functions in bone marrow due to bone marrow depression $(5,6)$. Apart from these reasons, the inhibitors in LC serum which include intestinal endotoxin, inflammatory cytokines, collagen, antibodies and other factors can affect the function of BM. We found in our preliminary experiments that the gradually accumulated LC related humoral inhibitors in vivo affected bone marrow endothelial cells (BMECs) and caused ultrastructural damage that was positively associated with the serious degree of LC (7).

The bone marrow microenvironment is the habitat of hematopoietic stem/progenitor cells (HSPCs) and it is where they proliferate and differentiate. BMECs are important components of the bone marrow microenvironment. They constitute a mechanical barrier between peripheral blood and the bone marrow microenvironment (8). Due to this special histological position, they not only function as cytoskeletal components supporting adhesion, migration and other activities of HSPCs, but also secrete cytokines to the bone marrow microenvironment $(9,10)$. A number of studies have demonstrated that these cytokines have important functions in regulating the directed homing, proliferation and differentiation of HSPCs (11-14). If BMECs are damaged, this results in dysfunction and the hematopoietic function of the bone marrow may therefore also be damaged. In addition, in a previous study, we found that the ultrastructure of BMECs was damaged by a humoral inhibitors in the sera of patients with LC (7).

Therefore, we hypothesized that the humoral inhibitors in the sera of patients with LC may induce damage and abnormal cytokine secretion by BMECs. This would then lead to a disturbance in the bone marrow microenvironment and the dysfunction of HSPCs. In order to verify this hypothesis and investigate what changes occur in BMECs and whether they abnormally secrete cytokines in LC, we cultured human BMECs with medium supplemented with $20 \%$ pooled sera from healthy volunteers or patients with LC, and the abnormally expressed signaling pathways and cytokines were screened using whole genome expression profiling, Human Cytokines RT ${ }^{2}$ Profiler $^{\mathrm{TM}}$ PCR array and in vitro experiments.

\section{Materials and methods}

Patients and serum collection. A total of 26 hospitalized patients (aged 35-62 years; mean age, 52.12 \pm 7.03 years) from the First Affiliated Hospital of Harbin Medical University, Harbin, China were selected to participate in this study from September 2011 to May 2012. The patients had been diagnosed with cholestatic LC and their liver functions were graded as Child-Pugh $\mathrm{A} / \mathrm{B}(\mathrm{A}=9, \mathrm{~B}=17)$. They were all male patients without viral hepatitis, alcoholic liver disease, other liver diseases and diseases in other systems, and peripheral blood samples were collected from the patients pior to the treatments. A total of 10 healthy male volunteers (aged 34-65 years; mean age, $52.90 \pm 8.39$ years) were selected after performing a physical examination, and their blood samples were collected to obtain the sera for the healthy control group. The study protocol was approved by the Ethics Committee of Harbin Medical University and informed consent was obtained from all patients, as per the guidelines of the World Medical
Association Declaration of Helsinki. Serum specimens were obtained by standard clinical laboratory processing with serum separated typically $<1 \mathrm{~h}$ after blood collection. Sera were heat inactivated for $40 \mathrm{~min}$ at $56^{\circ} \mathrm{C}$ and sterile filtered through $0.22-\mu \mathrm{m}$ filters (Millex, Millipore Corp., Bedford, MA, USA). After processing, the serum samples were stored in a refrigerator of $-20^{\circ} \mathrm{C}$ for further use, as previously described (15). Additionally, the data of the hematological parameters of the patients with LC and the healthy volunteers were obtained from the Medical Examination Center of the First Affiliated Hospital of Harbin Medical University (Table I).

Cell lines and cell culture conditions. Human BMECs (China Center for Type Culture Collection, Shanghai, China) were cultured in RPMI-1640 medium (Sigma, St. Louis, MO, USA) containing antibiotics $(100 \mathrm{U} / \mathrm{ml}$ of penicillin and $100 \mathrm{mg} / \mathrm{ml}$ of streptomycin) and incubated in $5 \% \mathrm{CO}_{2}$. In order to evaluate the effects of the humoral inhibitors in the sera of patients with LC on BMECs, this medium was modified to $20 \%$ pooled sera content with either the pooled sera of healthy controls $(n=10)$ or the pooled sera of patients with LC $(n=26)$; the other culture conditions were not changed. TRIzol reagent (Invitrogen Corp., Camarillo, CA, USA) was used to isolate the RNA after the human BMECs were treated for $48 \mathrm{~h}$, as previously described (15).

Apoptosis assay. After the BMECs were treated with medium supplemented with $20 \%$ pooled sera from patients with LC or healthy volunteers as the control group for $48 \mathrm{~h}, 1 \times 10^{6}$ cells were collected and washed twice with ice-cold PBS, suspended in binding buffer (100 $\mu \mathrm{l}$ ) (BD Biosciences, San Jose, CA, USA), treated with Annexin V and propidium iodide (PI) (BD Biosciences), and incubated in the dark for $15 \mathrm{~min}$; another $300 \mu \mathrm{l}$ binding buffer were then added and flow cytometry analysis was performed within $1 \mathrm{~h}$ to measure the rate of apoptosis (\%).

RNA extraction. Total RNA was extracted and isolated using TRIzol reagent (Invitrogen Corp.) following the manufacturer's instructions. RNA quantity and quality were measured using the NanoDrop ND-1000 spectrophotometer (NanoDrop Technologies, Inc., Wilmington, DE, USA). RNA integrity was assessed by standard denaturing agarose gel electrophoresis. For spectrophotometry, the OD A260/A280 ratio should be close to 2.0 for pure RNA (ratios between 1.8 and 2.1 are acceptable). The OD A260/A230 ratio should be $>1.8$.

Whole genome microarray analysis. The Human $12 \mathrm{x} 135 \mathrm{~K}$ Gene Expression array was manufactured by Roche NimbleGen (Madison, WI, USA). A total of 45,033 genes were collected from authoritative data sources, including NCBI. Double-stranded cDNA (ds-cDNA) was synthesized from total RNA using an Invitrogen SuperScript ds-cDNA synthesis kit in the presence of 100 pmol oligo(dT) primers. ds-cDNA was cleaned and labeled in accordance with the NimbleGen Gene Expression Analysis protocol. The purified cDNA was quantified using a NanoDrop ND-1000 spectrophotometer. For Cy3 labeling of cDNA, the NimbleGen One-Color DNA labeling kit was used according to the manufacturer's instructions. ds-cDNA $(1 \mu \mathrm{g})$ was incubated for $10 \mathrm{~min}$ at $98^{\circ} \mathrm{C}$ with $1 \mathrm{OD}$ of Cy3-9mer primer. Subsequently, 
Table I. Hematological parameters in the healthy group of volunteers and patients with liver cirrhosis (LC).

\begin{tabular}{lccccc}
\hline Group & $\mathrm{N}$ & Age & Hemoglobin $(\mathrm{g} / \mathrm{l})$ & Leukocytes (x10 $/ 1)$ & Platelets (x109/l) \\
\hline Healthy volunteers & 10 & $52.90 \pm 8.39$ & $146.8 \pm 6.8$ & $7.9 \pm 1.1$ & $213.6 \pm 20.2$ \\
Patients with LC & 26 & $52.12 \pm 7.03$ & $137.6 \pm 9.5$ & $6.8 \pm 1.9$ & $120.6 \pm 57.0^{\mathrm{a}}$ \\
\hline
\end{tabular}

Blood samples were obtained from 26 male patients with cholestatic LC and 10 male healthy volunteers. The results of hematological parameters were obtained from the Medical Examination Center of the First Affiliated Hospital of Harbin Medical University. Data are presented as the means \pm SD. The Student's t-test was used for differences between the experimental group and the control group ( $\left.{ }^{\mathrm{a}} \mathrm{p}<0.05\right)$.

Table II. Gene table for 84 important cytokine genes.

Category/family Genes

Interferons

Interleukins

Bone morphogenetic proteins (BMPs) and TGF-beta family

PDGF/VEGF family

TNF superfamily

Other growth factors/cytokines
IFNA1, IFNA2, IFNA4, IFNA5, IFNA8, IFNB1, IFNK, IFNG

IL10, IL11, IL12A, IL12B, IL13, IL14, IL15, IL16, IL17, IL17B, IL17C, IL17E, IL18, IL19, IL1A, IL1B, IL1F10, IL1F5, IL1F6, IL1F7, IL1F8, IL1F9, IL2, IL9, IL20, IL21, IL22, IL24, IL3, IL4, IL5, IL6, IL 7, IL8

BMP1, BMP2, BMP3, BMP4, BMP5, BMP6, BMP7, BMP8B, GDF10 (BMP3B), GDF11 (BMP11), GDF2 (BMP9), GDF3, GDF5, GDF8, GDF9, INHA, INHBA, NODAL, TGFA, TGFB1, TGFB2, TGFB3

FIGF (VEGFD), PDGFA

FASLG (TNFSF6), LTA (TNFB), LTB, TNF, TNFRSF11B, TNFSF13B, TNFSF10, TNFSF11, TNFSF12, TNFSF13, TNFSF13B, TNFSF14, TNFSF4, TNFSF7, TNFSF8

CSF1 (MCSF), CSF2 (GMCSF), LEFTY2 (EBAF), FAM3B

IFNA, interferon $\alpha$; IFNB, interferon beta; IFNG, interferon gamma; IFNK, interferon kappa; IL, interleukin; BMP, bone morphogenetic protein; TGFB, transforming growth factor-beta; TNF, tumor necrosis factor; LTA, lymphotoxin alpha; TNFB, tumor necrosis factor beta; CSF, colony-stimulating factor; TNFSF, tumor necrosis factor (ligand) superfamily; LEFTY2, left-right determination factor 2; FAM3B, family with sequence similarity 3 , member B; GDF, growth differentiation factor; INHA, inhibin alpha; FIGF, c-fos induced growth factor; PDGFA, platelet-derived growth factor alpha polypeptide; NODAL, nodal growth differentiation factor.

100 pmol of deoxynucleoside triphosphates and $100 \mathrm{U}$ of the Klenow fragment (New England Biolabs, Ipswich, MA, USA) were added and the mixture was incubated at $37^{\circ} \mathrm{C}$ for $2 \mathrm{~h}$. Microarrays were hybridized at $42^{\circ} \mathrm{C}$ for $16-20 \mathrm{~h}$ with $4 \mu \mathrm{g}$ of Cy3 labeled ds-cDNA in NimbleGen hybridization buffer/hybridization component A in a hybridization chamber (Hybridization System, NimbleGen Systems, Inc.). After being washed in an ozone-free environment, the slides were scanned using the Axon GenePix 4000B microarray scanner (Molecular Devices, LLC. Sunnyvale, CA, USA). Scanned images (TIFF format) were then imported into NimbleScan software (version 2.5) for grid alignment and expression data analysis. Expression data were normalized through quantile normalization and the Robust Multichip Average algorithm included in the NimbleScan software. All gene level files were imported into Agilent GeneSpring GX software (version 11.5.1; Agilent Technologies, Inc., Santa Clara, CA, USA) for further analysis. Differentially expressed genes were identified through fold change filtering.

Human Cytokines $R T^{2}$ Profiler $^{T M}$ PCR array. We used the Human Cytokines $\mathrm{RT}^{2}$ Profiler PCR array (SuperArray
Bioscience Corp., Frederick, MD, USA) according to the manufacturer's instructions to perform quantitative reverse transcription PCR (qRT-PCR) in the ABI PRISM 7900-HT machine (Applied Biosystems, Foster City, CA, USA). RNA isolation, DNase treatment and RNA clean-up were performed according to the manufacturer's instructions and as previously described (16). The Human Cytokines $\mathrm{RT}^{2}$ Profiler PCR array profiles the expression of 84 important cytokine genes. This array includes interferons (IFNs), interleukins (ILs), bone morphogenetic proteins (BMPs) and members of the transforming growth factor (TGF)B family. Also represented are platelet-derived and vascular endothelial growth factors. Tumor necrosis factors (TNFs) are included, as well as other cytokine-related genes (Table II). It also contains 12 internal control genes, including a panel of 5 housekeeping genes (B2M, HPRT1, RPL13A, GAPDH, ACTB), 3 reverse transcription controls, 3 positive PCR controls and human genomic DNA contamination (HGDC). After the preparatory detection, the expression of ACTB was comparatively constant, and we selected ACTB as the final control gene to control for variability in cDNA quantity, integrity and individual primer 
Table III. Primers used for quantitative PCR.

\begin{tabular}{lll}
\hline Gene & \multicolumn{1}{c}{ Forward primer } & \multicolumn{1}{c}{ Reverse primer } \\
\hline CCL28 & 5'-CAGAGAGGACTCGCCATCGT-3' & 5'-TGTGAAACCTCCGTGCAACA-3' \\
GDNF & 5'-CCAAAGCGTCCGAGACTG-3' & 5'-ACAAACGACCAAGACAGATCAGAG-3' \\
THBS2 & 5'-AGCTCGGCAGAGGCAGGATG-3' & 5'-ATACCATGCTCGATTTTCACTCC-3' \\
THBS3 & 5'-CTGCCTGCCAGGGGTAGTGAG-3' & 5'-TTAGAACTTTATGGCAACGTGCTG-3' \\
LIF & 5'-ACTTCCCGCCCTTCCACGC-3' & 5'-GGGAGGTGCCAAGGTACACGACT-3' \\
MST1 & 5'-GGTACGGGTAATGACACAGTCCTAAATG-3' & 5'-GTTGTGGGTAAAGCAGGCAAGTGG-3' \\
IGF1 & 5'-ACCGACATGCCCAAGACCCA-3' & 5'-TTCAGCATTTCTACTTCCAATCTCCCT-3' \\
MMP15 & 5'-CATGGAAACAACCTCTTCCT-3' & 5'-CTTGAAGTTGTCAACGTCCT-3' \\
MMP3 & 5'-CTGTTGATTCTGCTGTTGAG-3' & 5'-AAGTCTCCATGTTCTCTAACTG-3' \\
FGF22 & 5'-GGCCCCGCCCTTGTACTG-3' & 5'-TCAGGAGACCAGGACGGGCAG-3' \\
SLIT2 & 5'-CACCTCGTACAGCCGCACTT-3' & 5'-TGTGGACCGCTGAGGAGCAA-3' \\
CCL4L2 & 5'-ACAGGACACAGCTAGGTTC-3' & 5'-GCATTTTATTTTAAAGTTTTATTATG-3' \\
VTN & 5'-GAGCAAACAGAGCAGCAGAAAAG-3' & 5'-TTTAAACTCGGGGCTAAGGGACC-3' \\
GAPDH & 5'-TCAATGACAACTTTGTCAAGCTCA-3' & 5'-GTGGGTGGTCCAGGGTTTCTTACT-3' \\
\hline
\end{tabular}

efficiency in the whole RT-PCR reaction, and presented the data normalized to ACTB expression. Data normalization was based on correcting all $\mathrm{Ct}$ values for the average $\mathrm{Ct}$ values of several constantly expressed housekeeping genes present on the array, as previously described (17).

$q R T-P C R$. To validate the whole genome microarray data, qRT-PCR was performed using the $S_{Y B R}{ }^{\circledR}$ PrimeScript $^{\mathrm{TM}}$ RT-PCR kit in the LightCycler System (Roche Diagnostics, Lewes, UK) for 13 supplementary cytokine genes. Samples were selected from the original microarray experiments for further qRT-PCR testing based on sufficient RNA remaining. Total RNA was extracted from the serum samples using TRIzol reagent (Invitrogen Corp.) containing RNAsafe (Tiangen Biotech Co., Ltd., Beijing, China) and converted to first-strand cDNA according to the manufacturer's instructions. Glyceraldehyde-3-phosphate dehydrogenase (GAPDH) expression was used as an internal control and the relative expression of each gene was determined by the fold change $\left(2^{-\Delta \Delta C t}\right)$ method, comparing the expression of the test gene to an average GAPDH, and then comparing the LC group versus the healthy control group. The primers used for cDNA amplification are presented in Table III.

Statistical analysis. Patient data are expressed as the means \pm standard deviation (SD). Comparisons were made using the Student's t-test. In the process of screening differentially expressed genes in the whole genome microarray and the Human Cytokines $\mathrm{RT}^{2}$ Profiler PCR array, a $\geq 2$-fold change indicated a significant change in gene expression based on the microarray manufacturer's recommendations. Categorical variables are given as numbers and percentages; Fisher's exact test was used to test their associations. Statistical analyses were set with a significance level of 0.05 and were performed using SPSS 15.0 statistical software (SPSS Inc., Chicago, IL, USA).

\section{Results}

Whole genome microarray analysis. We investigated the gene expression profiles in BMECs treated with sera from patients with LC or sera from healthy volunteers using whole genome microarray. To identify differentially expressed genes, we performed a fold change filtering between the 2 samples. The threshold is a fold change $\geq 2.0$ or $\leq-2.0$. The results revealed that there were 1,106 upregulated genes and 766 downregulated genes in the BMECs treated with sera from patients with LC. Only genes with $\mathrm{p}<0.05$ and a fold change $\geq 2.0$ for upregulation and $\leq-2.0$ for downregulation were considered for expression analysis.

Gene Ontology $(G O)$ analysis and signaling pathway analysis of differentially expressed genes. In order to identify the activated pathways present in BMECs treated with sera from patients with LC, functional categories of the differentially expressed genes were identified using GO analysis. The GO categories are derived from Gene Ontology (www.geneontology.org), which comprises 3 structured networks of defined terms to describe gene product attributes. An enrichment score which is equal to $-\log 10 \mathrm{p}$-value denotes the significance of GO term enrichment in the differentially expressed gene list. The lower the enrichment score is, the more significant the GO term $(\mathrm{p}<0.05)$. The most significant functional categories of the genes were as follows: a large number of upregulated genes were involved in processes, such as cell-cell adhesion, apoptosis and cellular response to stimuli and the downregulated genes were involved in the negative regulation of secretion, angiogenesis, blood vessel development and cell growth (Fig. 1A).

Based on the latest Kyoto Encyclopedia of Genes and Genomes (KEGG) database, we carried out pathway analysis of the differentially expressed genes. The results revealed the pathways with the highest enrichment scores as follows: the upregulated genes were either cell adhesion molecules, 


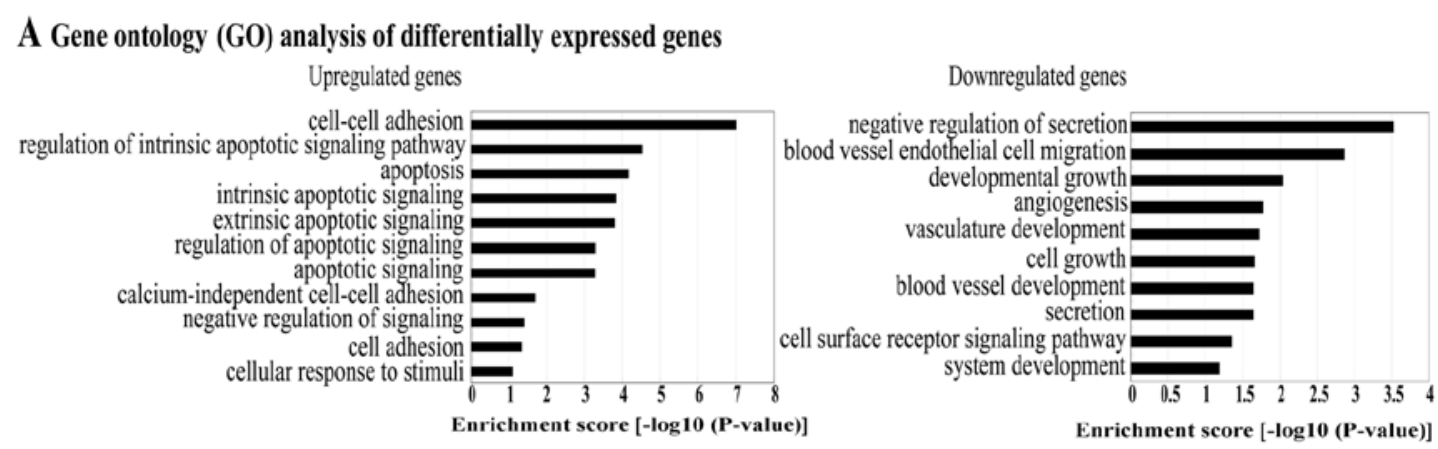

B Pathway analysis of differentially expressed genes

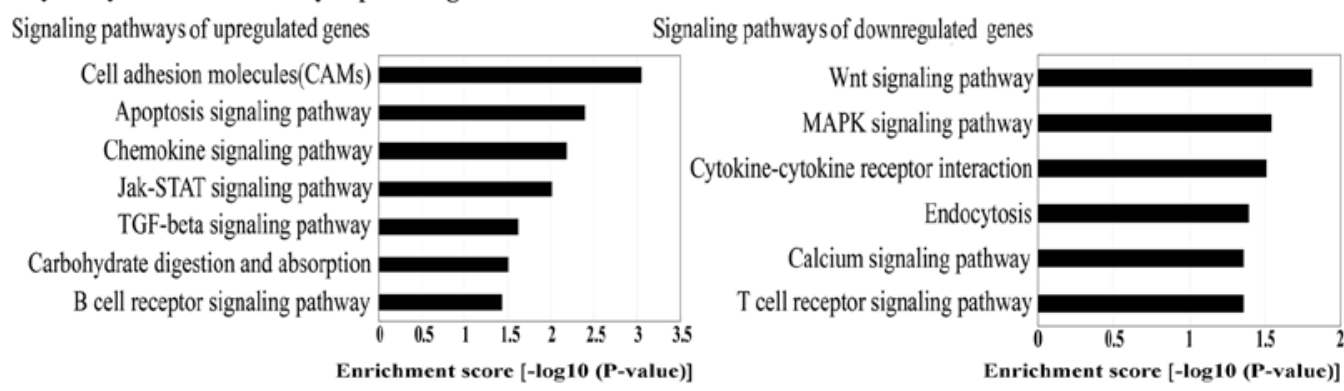

Figure 1. Functional grouping and signaling pathways of upregulated and downregulated genes in bone marrow endothelial cells (BMECs) treated with sera from patients with liver cirrhosis (LC) for $48 \mathrm{~h}$. (A) All the gene identifications were examined for their known biological functions according to the Gene Ontology Consortium and grouped in the respective functional category. (B) Pathway analysis of differentially expressed genes was carried out based on the latest data from the KEGG database. Significance is expressed as a p-value calculated using Fisher's exact test $(\mathrm{p}<0.05)$.
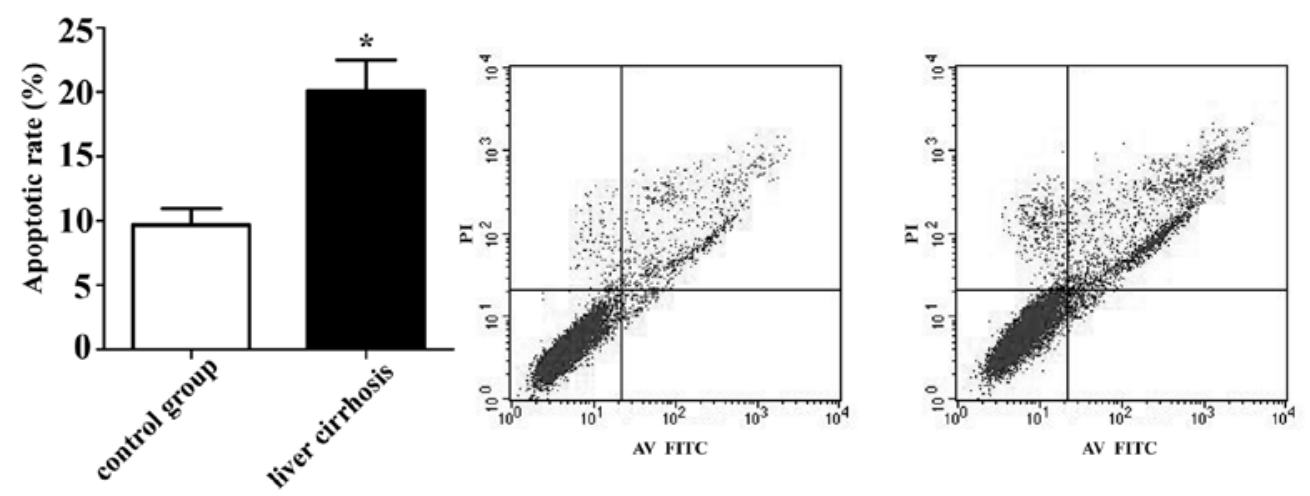

Figure 2. Apoptosis was induced in bone marrow endothelial cells (BMECs) treated with sera from patients with liver cirrhosis (LC). The results of flow cytometry analysis revealed that the BMECs treated with sera from patients with LC had a higher apoptotic percentage (20.12 $\pm 2.39 \%)$ compared with the control group $(9.68 \pm 1.28 \%)$ following cultured for $48 \mathrm{~h}(\mathrm{p}<0.05)$.

or involved in the chemokine signaling pathway, Jak-STAT signaling pathway, TGFB signaling pathway, carbohydrate digestion and absorption and the B cell receptor signaling pathway. The downregulated genes were involved in the Wnt signaling pathway, MAPK signaling pathway, cytokine-cytokine receptor interaction, endocytosis, calcium signaling pathway and the $\mathrm{T}$ cell receptor signaling pathway (Fig. 1B). The upregulated genes were either cell adhesion molecules, or involved in the apoptotic signaling pathway (Table IV) and the downregulated genes were involved in the Wnt signaling pathway and MAPK signaling pathway (Table V) which had the highest enrichment scores and greatest statistical significance $(\mathrm{p}<0.05)$.

Apoptosis assay of BMECs treated with sera from patients with $L C$. The results of flow cytometry analysis revealed that the BMECs treated with sera from patients with LC had a higher percentage of apoptosis $(20.12 \pm 2.39 \%)$ compared with the control group $(9.68 \pm 1.28 \%)$ following cultured for $48 \mathrm{~h}(\mathrm{p}<0.05)$ (Fig. 2). These results suggest that serum from patients with LC induces BMEC apoptosis, which confirms the accuracy of bioinformatic analysis.

Human Cytokines $R T^{2}$ Profiler PCR array. In order to investigate the cytokines abnormal expressed by human BMECs following treatment with the pooled sera from patients with LC for 48 h, the Human Cytokines RT ${ }^{2}$ Profiler PCR array was carried out to detect the mRNA expression of 84 important cytokines. The typical amplification curves from the sample of BMECs treated with sera from patients with LC analyzed by RT-PCR are shown in Fig. 3. To identify the differen- 
Table IV. Pathways of upregulated genes

\begin{tabular}{|c|c|c|c|c|}
\hline Gene name & $\begin{array}{c}\text { Gene } \\
\text { ID }\end{array}$ & Description & $\begin{array}{c}\text { Fold } \\
\text { change }\end{array}$ & Function (GO analysis) \\
\hline \multicolumn{5}{|c|}{ Cell adhesion molecules } \\
\hline ALCAM & 214 & Activated leukocyte cell adhesion molecule & 2.11 & Cell adhesion \\
\hline ICAM1 & 3383 & Intercellular adhesion molecule 1 & 3.18 & Cell-cell adhesion \\
\hline $\mathrm{CDH} 1$ & 999 & Cadherin 1, type 1, E-cadherin & 2.41 & Cell adhesion \\
\hline $\mathrm{CDH} 3$ & 1001 & Cadherin 3 , type 1 , P-cadherin & 4.66 & Cell adhesion, response to stimuli \\
\hline CDH5 & 1003 & Cadherin 5, type 2, VE-cadherin & 2.39 & Cell adhesion \\
\hline CLDN1 & 9076 & Claudin 1 & 2.00 & Calcium-independent cell-cell adhesion \\
\hline CLDN14 & 23562 & Claudin 14 & 2.35 & Calcium-independent cell-cell adhesion \\
\hline CLDN3 & 1365 & Claudin 3 & 2.13 & Calcium-independent cell-cell adhesion \\
\hline CLDN4 & 1364 & Claudin 4 & 2.04 & Calcium-independent cell-cell adhesion \\
\hline CLDN7 & 1366 & Claudin 7 & 3.11 & Calcium-independent cell-cell adhesion \\
\hline CLDN9 & 9080 & Claudin 9 & 2.44 & Calcium-independent cell-cell adhesion \\
\hline SELPLG & 6404 & Selectin P ligand & 3.17 & Cell adhesion \\
\hline HLA-DQB1 & 3119 & Major histocompatibility complex, class II, DQ beta 1 & 2.11 & Cell adhesion \\
\hline HLA-DRB1 & 3123 & Major histocompatibility complex, class II, DR beta 1 & 2.15 & Cell adhesion, immune response \\
\hline ICOSLG & 23308 & Inducible T-cell co-stimulator ligand & 4.26 & Cell adhesion, defense response \\
\hline NCAM2 & 4685 & Neural cell adhesion molecule 2 & 2.67 & Cell adhesion \\
\hline PVRL2 & 5819 & Poliovirus receptor-related 2 & 2.04 & Cell adhesion \\
\hline \multicolumn{5}{|c|}{ Apoptotic signaling pathway } \\
\hline AKT1 & 207 & v-akt murine thymoma viral oncogene homolog 1 & 2.68 & Cell apoptosis \\
\hline PIK3R 1 & 5295 & Phosphoinositide-3-kinase, regulatory subunit 1 & 2.18 & Immune response \\
\hline PIK3R2 & 5296 & Phosphoinositide-3-kinase, regulatory subunit 2 & 2.15 & Negative regulation of anti-apoptosis \\
\hline PIK3R3 & 8503 & Phosphoinositide-3-kinase, regulatory subunit 3 & 3.05 & Response to stimuli \\
\hline DFFB & 1677 & DNA fragmentation factor, beta polypeptide & 2.52 & Cell apoptosis \\
\hline MYD88 & 4615 & Myeloid differentiation primary response gene (88) & 5.12 & Cell apoptosis \\
\hline PRKACA & 5566 & Protein kinase, cAMP-dependent, catalytic, alpha & 3.47 & Protein amino acid phosphorylation \\
\hline TRADD & 8717 & TNFRSF1A-associated via death domain & 2.22 & Cell apoptosis \\
\hline
\end{tabular}

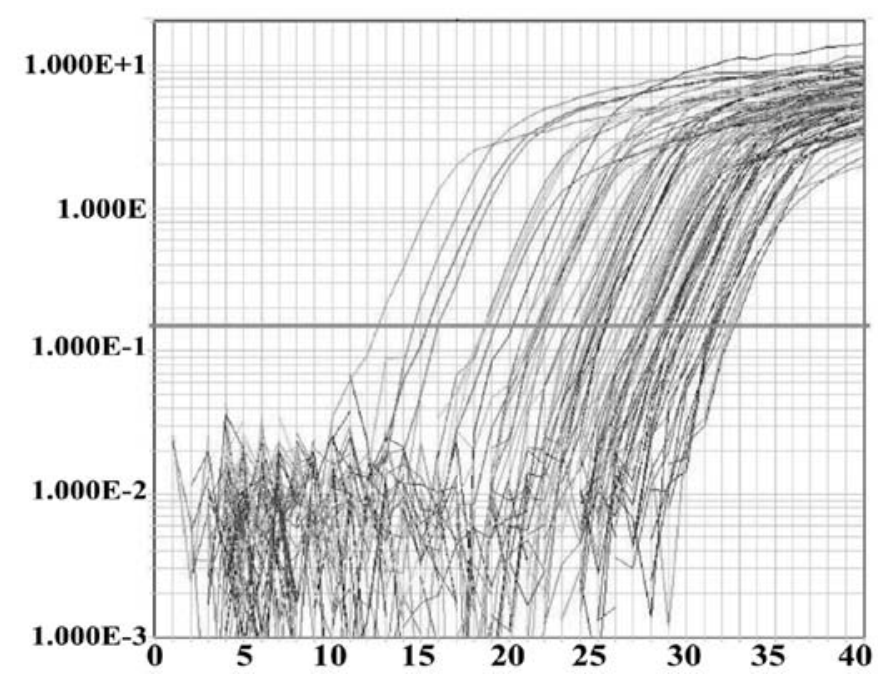

Figure 3. Amplification curve of a sample with the RT-PCR array. A typical amplification curve from a sample of BMECs treated with sera from patients with LC in the Human Cytokines RT ${ }^{2}$ Profiler $^{\mathrm{TM}}$ PCR array. tially expressed genes, we performed fold change filtering between the 2 samples. The threshold is a fold change $\geq 2.0$ or $\leq-2.0$. The results revealed that TGFB1, TNFB, IL24 and TNF receptor superfamily, member $11 \mathrm{~b}$ (TNFRSF11B) were upregulated (fold change $\geq 2.0$ ) and IL1A, IL6, IL11, IL17C, family with sequence similarity 3 , member B (FAM3B), Fas ligand (FASLG) and TNF (ligand) superfamily, member 13b (TNFSF13B) were downregulated (fold change $\leq-2.0$ ) in the BMECs treated with sera from patients with LC (Fig. 4).

Supplementary screening and validating of cytokine genes by whole genome microarray analysis and $q R T-P C R$. In order to detect more differentially expressed cytokine genes in the BMECs treated with sera from patients with LC, we also screened 13 genes (cytokines, chemokines and growth factors) which were not identified as differentially expressed genes in the whole genome microarray (Table VI). In order to confirm the accuracy of the results, qRT-PCR was carried out to validate the 13 genes. The results revealed that 10 of the 13 differently expressed genes were consistent with the data from microarray 
Table V. Pathways of downregulated genes.

\begin{tabular}{|c|c|c|c|c|}
\hline Gene name & $\begin{array}{c}\text { Gene } \\
\text { ID }\end{array}$ & Description & $\begin{array}{l}\text { Fold } \\
\text { change }\end{array}$ & Function (GO analysis) \\
\hline \multicolumn{5}{|c|}{ Wnt signaling pathway } \\
\hline CSNK2A1 & 1457 & Casein kinase 2 , alpha 1 polypeptide & -2.06 & Regulation of signaling \\
\hline PLCB2 & 5330 & Phospholipase C, beta 2 & -3.10 & Signal transduction \\
\hline PORCN & 64840 & Porcupine homolog & -2.80 & Signal transduction \\
\hline РPP3CA & 5530 & Protein phosphatase 3 , catalytic subunit, alpha isoform & -2.34 & Cellular developmental process \\
\hline PPP3R1 & 5534 & Protein phosphatase 3 , regulatory subunit $\mathrm{B}$, alpha isoform & -2.05 & Blood vessel development \\
\hline PPP3R2 & 5535 & Protein phosphatase 3 , regulatory subunit $\mathrm{B}$, beta isoform & -2.59 & Blood vessel development \\
\hline PSEN1 & 5663 & Presenilin 1 & -2.17 & Cellular developmental process \\
\hline TCF7 & 6932 & Transcription factor 7 & -2.25 & Cellular developmental process \\
\hline WNT11 & 7481 & Wingless-type MMTV integration site family, member 11 & -2.02 & Blood vessel development \\
\hline WNT3 & 7473 & Wingless-type MMTV integration site family, member 3 & -2.12 & Cellular developmental process \\
\hline \multicolumn{5}{|c|}{ MAPK signaling pathway } \\
\hline CACNA1G & 8913 & Calcium channel, voltage-dependent, alpha $1 \mathrm{G}$ subunit & -2.62 & Cellular developmental process \\
\hline DUSP6 & 1848 & Dual specificity phosphatase 6 & -2.14 & Developmental growth \\
\hline DUSP8 & 1850 & Dual specificity phosphatase 8 & -3.23 & Signal transduction \\
\hline EGFR & 1956 & Epidermal growth factor receptor & -2.01 & Signal transduction \\
\hline FGF22 & 27006 & Fibroblast growth factor 22 & -2.09 & Cellular developmental process \\
\hline IL1A & 3552 & Interleukin 1, alpha & -2.04 & Blood vessel development \\
\hline MOS & 4342 & v-mos Moloney murine sarcoma viral oncogene homolog & -15.86 & Cell cycle \\
\hline NF1 & 4763 & Neurofibromin 1 & -2.91 & Blood vessel development \\
\hline NTRK1 & 4914 & Neurotrophic tyrosine kinase, receptor, type 1 & -4.27 & Blood vessel development \\
\hline PLA2G2F & 64600 & Phospholipase A2, group IIF & -2.73 & Cellular lipid metabolic process \\
\hline RASGRP1 & 10125 & RAS guanyl releasing protein 1 & -2.61 & Cellular lipid metabolic process \\
\hline
\end{tabular}

Table VI. Supplementary analysis of differentially expressed genes (cytokines, chemokines and growth factors) in the whole genome microarray.

\begin{tabular}{lrcl}
\hline Gene name & Gene ID & Fold change & \multicolumn{1}{c}{ Gene description } \\
\hline Upregulation & & & \\
CCL28 & 56477 & 2.46 & Chemokine ligand 28 \\
GDNF & 2668 & 2.16 & Glial cell derived neurotrophic factor \\
THBS2 & 7058 & 3.11 & Thrombospondin 2 \\
LIF & 3976 & 2.32 & Leukemia inhibitory factor \\
THBS3 & 7059 & 2.01 & Thrombospondin 3 \\
MST1 & 4485 & 3.02 & Macrophage stimulating 1 \\
Downregulation & & & \\
MMP15 & 4324 & -2.46 & Matrix metallopeptidase 15 \\
FGF22 & 27006 & -2.09 & Fibroblast growth factor 22 \\
MMP3 & 4314 & -2.22 & Matrix metallopeptidase 3 \\
SLIT2 & -2.03 & Slit homolog 2 \\
IGF1 & 9353 & -2.12 & Insulin-like growth factor 1 \\
CCL4L2 & 3479 & -3.63 & Chemokine ligand 4-like 2 \\
VTN & 388372 & -2.41 & Vitronectin \\
\hline
\end{tabular}

analysis. The fold changes of those 10 gene expressions, as determined by whole genome microarray and qRT- PCR, are presented in Fig. 5.
Differentially expressed cytokine genes and bone marrow haemopoiesis. The Human Cytokines RT ${ }^{2}$ Profiler PCR array, whole genome microarray and qRT-PCR were carried out to 


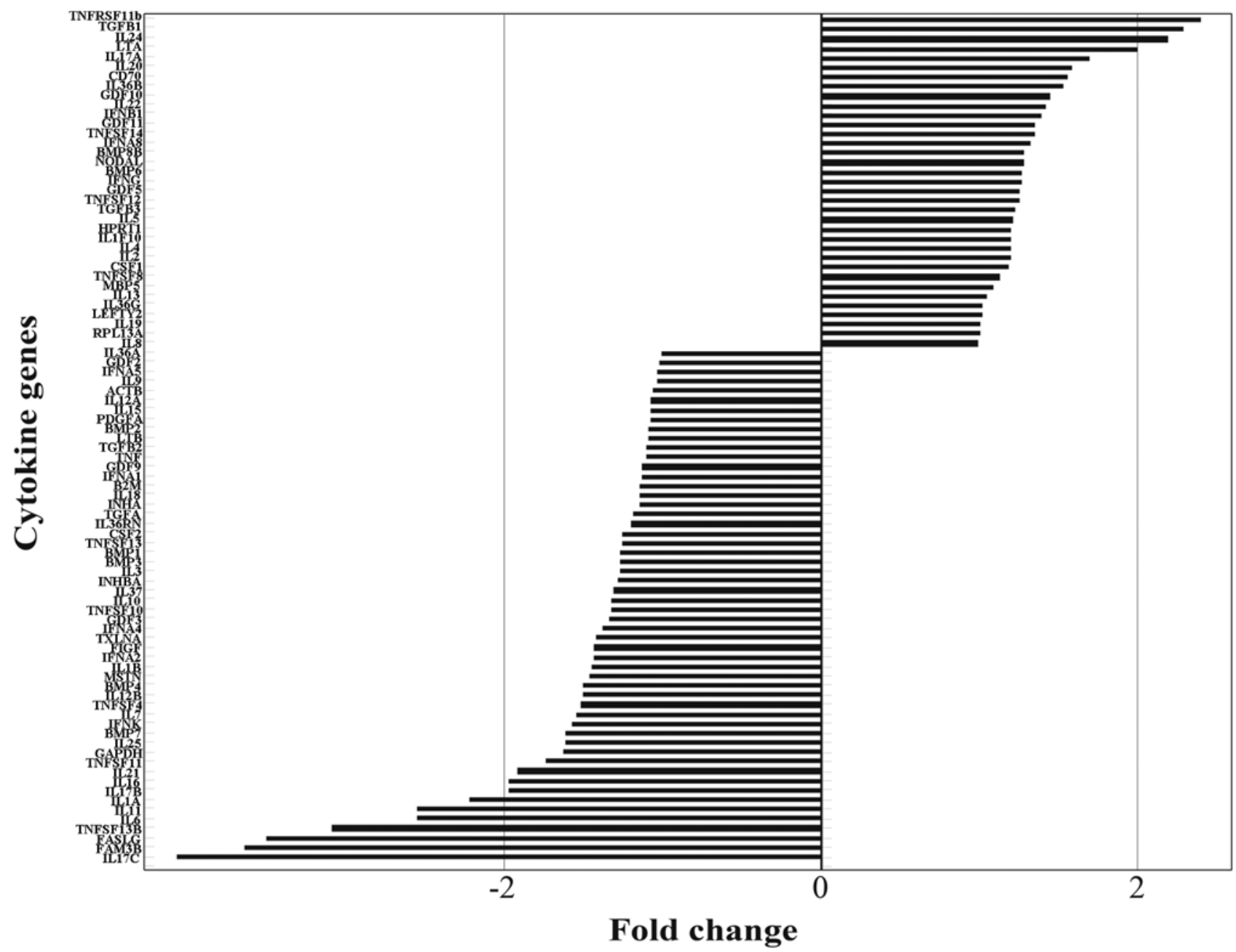

Figure 4. The results of human cytokines $\mathrm{RT}^{2}$ Profiler $^{\mathrm{TM}}$ PCR array. The array was carried out to detect the mRNA expression of 84 important cytokines in BMECs treated with sera from healthy volunteers or from patients with LC serum. A fold change filtering was performed between the 2 samples to identify differentially expressed genes. The threshold was a fold ahange $\geq 2.0$ or $\leq-2.0$. The results indicated that TGFB1, TNFB (LTA), IL24 and TNFRSF11B were upregulated (fold change $\geq 2.0$ ) and IL1A, IL6, IL11, IL17C, FAM3B, FASLG and TNFSF13B were downregulated (fold change $\leq-2.0$ ) in the BMECs treated with sera from patients with LC.

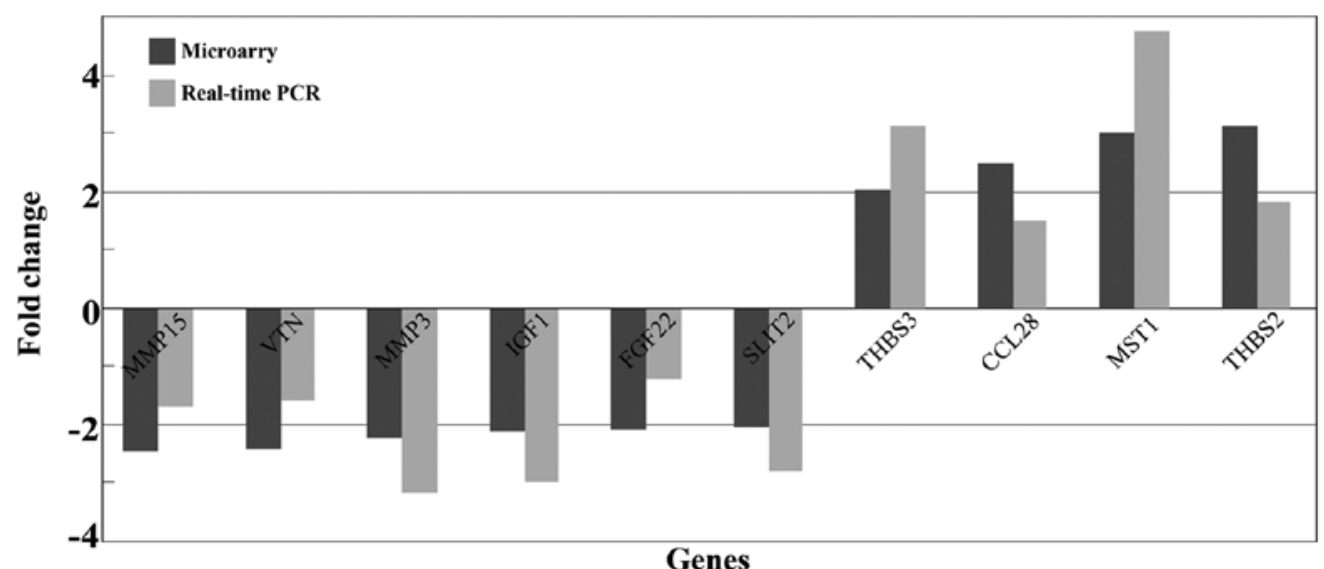

Figure 5. Concordance in the expression of the supplementary cytokine genes between microarray and qRT-PCR. The mRNA expression of supplementary cytokine genes from the microarray data was confirmed by qRT-PCR. The results revealed that the data of 10 genes of the 13 differently expressed genes were consistent with the data from microarray analysis. Data are presented as fold changes in BMECs treated with sera from patients with LC as compared with the BMECs treated with sera from healthy volunteers. 
factor 1 (IGF1), fibroblast growth factor 22 (FGF22), slit homolog 2 (Drosophila) (SLIT2), thrombospondin (THBS)2, THBS3, chemokine (C-C motif) ligand 28 (CCL28) and macrophage stimulating 1 (MST1)] from 97 cytokine genes in the BMECs treated with sera from patients with LC. After retrieving a large number of literature on the functions of the 21 differentially expressed cytokines during bone marrow hemopoiesis in PubMed and then analyzing these results, we found that the hemopoiesis-stimulating factors, IL1A, IL6, IL11 and IGF1 were downregulated, whereas the hemopoiesis-inhibitory factors, TGFB1, TNFB and MST1 were upregulated.

\section{Discussion}

LC refers to multiple organ and system disorder, including the kidneys, brain and other organs induced by extensive inflammatory reactions and damage to hepatic functions (18). Clinical trials and experimental studies have shown that the humoral inhibitors in the sera of patients with LC can affect hematopoietic tissue by acting on erythroid differentiation (19). In our previous study, in vivo experiments revealed that the important components of the bone marrow microenvironment (BMECs) in patients with LC and mice with LC underwent changes after CCL4 induction (7). In order to further investigate the effects of LC on BMECs, in this study, we carried out whole genome expression profiling to detect differentially expressed genes in human BMECs treated with pooled sera from patients with LC or sera from healthy volunteers as the control group. The results revealed that 1,106 genes were upregulated and 766 genes were downregulated. The results from GO analysis and pathway analysis indicated that the important upregulated genes were involved in processes such as cell adhesion, cellular response to stimuli and cell apoptosis and these genes were mainly either cell adhesion molecules or involved in the apoptotic pathway; the important downregulated genes were involved in blood vessel endothelial cell migration, cell surface receptor signaling, vasculature development and secretion and these genes mainly belonged to the Wnt and MAPK pathways. Moreover, apoptosis assay confirmed that the humoral inhibitors in the sera of patients with LC induced the apoptosis of BMECs.

The pathway of cell adhesion molecules involves the high expression of selectin P ligand (SELPLG), intercellular adhesion molecule 1 (ICAM1), cadherin (CDH)1, CDH3, CDH5 and other cell adhesion genes. SELPLG is the most wellinvestigated P-selectin glycoprotein ligand and it belongs to the adhesion molecule family for granulocytes, platelets and endothelial cell adhesion, which can promote the incidence of inflammatory reactions and vascular lesions $(20,21)$. ICAM1 is expressed at relatively low levels in normal BMECs and its overexpresion indicates damage or the activation of endothelial cells (21-23). CDH1, CDH3 and CDH5 belong to the cadherin family, among which CDH5 (VE-cadherin) plays important roles in regulating the stability and integrity of the vascular endothelium (24). It can coordinate some signals, inhibit the growth of vascular cells and decrease their permeability (25). The overexpression of these adhesion molecules indicates that the blood pathogenic factors of LC may lead to changes in BMECs.
The expression levels of protein kinase, cAMP-dependent, catalytic, alpha (PRKACA or PKA), DNA fragmentation factor, $40 \mathrm{kDa}$, beta polypeptide (caspase-activated DNase) (DFFB), myeloid differentiation primary response 88 (MYD88), TNFRSF1A-associated via death domain (TRADD) and other genes in the apoptotic pathway increased. PKA can function in promoting endothelial cell apoptosis and inhibiting vascularization. Kim et al found that PKA inhibition can promote vascular endothelial formation (26). DFFB is a type of nucleotidase expressed during genomic DNA fragmentation in cell apoptosis. The overexpression of DFFB indicates an increase in apoptosis (27). MYD88 negatively regulates cell growth in several hematopoietic and non-hematopoietic cell types, including growth inhibition and apoptosis (28). TRADD can continuously recruit TNF, Fas and other cytokines to induce cell death (29). More importantly, the results of flow cytometry analysis suggested that sera from patients with LC induced BMEC apoptosis; these results ares consistent with the results of bioinformatic analysis.

The downregulated genes, such as wingless-type MMTV integration site family, member 3 (Wnt3), wingless-type MMTV integration site family, member 11 (Wnt11) and presenilin (PSEN) are involved in the Wnt pathway. The Wnt pathway plays important roles in cell formation and angiogenesis. Wnt 3 can regulate endothelial cell proliferation and angiogenesis by inducing Nanog transcription (30). Zhou et al found that Wnt11 played important roles in regulating cardiac formation. Wnt11 signaling can regulate the morphogenesis of the cardiac outflow tract by affecting extracellular matrix formation, cytoskeletal reorganization and polarized cell migration (31). PSEN1 can regulate the proliferation and differentiation of endothelial progenitor cells, and the functional loss of PSEN1 in the endothelial cell lineage may induce vascular diseases (32). The downregulation of these angiogenesis-promoting genes in the Wnt pathway indicates that the humoral inhibitors in the sera of patients with LC may affect the capacity of BMECs for angiogenesis.

Epidermal growth factor receptor (EGFR), calcium channel, voltage-dependent, $\mathrm{T}$ type, alpha $1 \mathrm{G}$ subunit (CACNA1G), v-mos Moloney murine sarcoma viral oncogene homolog (Mos) and other genes in the MAPK pathway are expressed at low levels. The MAPK pathway is associated with promoting vascular endothelial proliferation and angiogenesis. EGFR plays a key role in maintaining the functions of endothelial cells and endothelial cells are unable to grow after the knockout of the EGFR gene (33). Angiotensin II can induce CACNA1G expression by the AT1 receptor, Ras and MEK in endothelial cells, and CACNA1G can mediate angiotensin II to promote endothelial cell migration (34). Mos is a type of proto-oncogene encoding serine and tyrosine kinases and it can regulate the cell cycle in meiosis (35). The low expression levels of these genes in the MAPK pathway indicates that the pathogenic factors of LC may affect the angiogenic capacity of BMECs and cell growth.

In addition to the above, a more important result was found in our experiments. The Human Cytokines $\mathrm{RT}^{2}$ Profiler PCR array, whole genome microarray analysis and qRT-PCR were carried out to screen and verify 21 differentially expressed cytokine genes from 97 detected cytokine genes in BMECs following treatment for $48 \mathrm{~h}$ with pooled sera from patients with 
LC. Of note, further analysis on these differentially expressed genes indicated that the positive hematopoietic factors, IL1A, IL6, IL11 and IGF1, were expressed at a low levels, while the negative hematopoietic factors, TGFB1, TNFB and MST1, were expressed at high levels.

As shown above, BMECs are extremely important components of the bone marrow microenvironment, and they constitute a barrier between the bone marrow microenvironment and peripheral blood circulation (8). They are in direct contact with blood and are exposed to the environment with pathogenic factors for LC; on the other hand, they function as cytoskeletal components to support the adhesion of HSPCs in the bone marrow microenvironment (9). More importantly, they secrete cytokines to the bone marrow microenvironment by paracrine secretion, which regulates positioning, homing, proliferation and the differentiation of HSPCs (10). The results of the Human Cytokines RT ${ }^{2}$ Profiler PCR array and the whole genome microarray revealed that the positive hematopoietic factors, ILA1, IL6, IL11 and IGF1, secreted by BMECs were expressed at low levels following treatment with pooled sera from patients with LC; these factors all have functions in promoting hematopoiesis; for instance, IL1A has several functions, and it can regulate hematopoiesis by inducing the release of other active molecules or coordinating thrombopoietin (TPO) (36). As for IL6, Bernad et al found that IL6 can promote growth and the self-renewal of hematopoietic stem cells and early hematopoietic progenitor cells (11). In vivo and in vitro experiments have shown that IL11 can exert effects on several types of hematopoietic cells, and previous studies have shown that the in vivo hematopoietic functions of IL11 are significant, and that it can significantly stimulate the platelet count in the peripheral blood of normal animals or animals with bone marrow depression (12). Although IGF1 is not a classical hematopoietic growth factor, it can promote the maturation of erythroid cells and the proliferation of erythroid progenitor cells. Furthermore, IGF1 can promote heme synthesis and acidophilic erythroblast enucleation (37).

Moreover, we also found that the mRNA levels of the negative hematopoietic factors, TGFB1, TNFB and MST1, were expressed at high levels following treatment with sera from patients with LC; these factors have functions in inhibiting hematopoiesis. For example, TGFB1 is not only an important mediator of LC, but also a negative hematopoietic regulatory factor which can directly inhibit the proliferation of early hematopoietic stem cells $(13,38)$. As for TNFB, Skobin et al found that TNFB can inhibit the growth of erythroid cells, granulocytes and megakaryoblasts (39). Broxmeyer et al (40) reported that MST1 can coordinate with stimulating factors for early hematopoietic progenitor cells and significantly inhibit the proliferation of early hematopoietic progenitor cells. Cheng et al found that MST1 secreted by BMECs had significant inhibitory activities on hematopoietic progenitor cells (41).

In conclusion, adhesion molecules and apoptotic pathways were expressed at high levels in BMECs treated with sera from patients with LC, and the Wnt pathway and MAPK pathway, which promote vascularization and endothelial cell proliferation were expressed at low levels. Moreover, apoptosis was induced in the BMECs treated with sera from patients with LC. These results indicate that the humoral inhibitors in the sera of patients with LC may damage BMECs, affect their growth and induce cell apoptosis. Moreover, the positive hemopoietic factors secreted by BMECs treated with sera from patients with LC were downregulated, and the negative hemopoietic factors were upregulated, which may lead to a disturbance in the bone marrow microenvironment and the dysfunction of HSPCs, finally causing abnormalities in hematological indices in patients with LC. This phenomenon may be one of the important reasons for hematological abnormalities in patients with LC, and may provide novel therapeutic targets for scientific researches and clinical trials in the future.

\section{Acknowledgements}

This study was supported by a grant from the National Natural Science Foundation of China (811170397).

\section{References}

1. Qamar AA, Grace ND, Groszmann RJ, et al: Incidence, prevalence and clinical significance of abnormal hematological indices in compensated cirrhosis. Clin Gastroenterol Hepatol 7: 689-695, 2009.

2. Qamar AA and Grace ND: Abnormal hematological indices in cirrhosis. Can J Gastroenterol 23: 441-445, 2009.

3. Rois R, Sangro B, Herrero I, et al: The role of thrombopoietin in the thrombocytopenia of patients with live cirrhosis. Am J Gastroenterol 100: 1131-1136, 2005.

4. Sheehy T and Berman A: The anemia of cirrhosis. J Lab Clin Med 56: 72-82, 1960.

5. Sullivan LW and Herbert V: Suppression of hematopoiesis by ethanol. J Clin Invest 43: 2048-2062, 1964.

6 . Rosenfeld SJ and Young NS: Viruses and bone marrow failure. Blood Rev 5: 71-77, 1991

7. Zhao S, Fu YM, Zhang WH, et al: Alterations of bone marrow sinusoidal endothelium in rat and patients with liver cirrhosis. Dig Dis Sci 55: 654-661, 2010.

8. Kopp HG, Avecilla ST, Hooper AT and Rafii S: The bone marrow vascular niche: home of HSC differentiation and mobilization. Physiology 20: 349-356, 2005.

9. Rafii S, Shapiro F, Rimarachin J, et al: Isolation and characterization of human bone marrow microvascular endothelial cells: hematopoietic progenitor cell adhesion. Blood 84: 10-19, 1994.

10. Li WM, Huang WQ, Wang QR, et al: Positive and negative haematopoietic cytokines produced by bone marrow endothelial cells. Cytokine 12: 1017-1023, 2000.

11. Bernad A, Kopf M, Kulbacki R, et al: Interleukin-6 is required in vivo for the regulation of stem cells and committed progenitors of the hematopoietic system. Immunity 1: 725-731, 1994.

12. Schwertschlag US, Trepicchio WL, Dykstra KH, et al: Hematopoietic, immunomodulatory and epithelial effects of interleukin-11. Leukemia 13: 1307-1315, 1999.

13. Sitnicka E, Ruscetti FW, Priestley GV, et al: Transforming growth factor beta 1 directly and reversibly inhibits the initial cell divisions of long-term repopulating hematopoietic stem cells. Blood 88: 82-88, 1996.

14. Fossati G, Mazzucchelli I, Gritti D, et al: In vitro effects of GM-CSF on mature peripheral blood neutrophils. Int J Mol Med 1: 943-951, 1998.

15. Kramann R, Couson SK, Neuss S, et al: Uraemia disrupts the vascular niche in a 3D co-culture system of human mesenchymal stem cells and endothelial cells. Nephrol Dial Transplant 27: 2693-2702, 2012

16. Tjandra SS, Hsu C, Goh YI, et al: IFN-beta signaling positively regulates tumorigenesis in aggressive fibromatosis, potentially by modulating mesench-ymal progenitors. Cancer Res 67: 7124-7131, 2007.

17. Livak KJ and Schmittgen TD: Analysis of relative gene expression data using real-time quantitative PCR and the 2(-Delta Delta C(T)) Method. Methods 25: 402-408, 2001.

18. Bataller R and Brenner DA: Liver fibrosis. J Clin Invest 115: 209-218, 2005.

19. Ruhenstroth-Bauer G: The role of humoral splenic factors in the formation and release of blood cells. Semin Hematol 2: 229-248, 1965. 
20. Kansas GS: Selectins and their ligands: current concepts and controversies. Blood 88: 3259-3287, 1996.

21. Wagner DD and Frenette PS: The vessel wall and its interactions. Blood 111: 5271-5281, 2008.

22. Schweitzer CM, van der Schoot CE, Drager AM, et al: Isolation and culture of human bone marrow endothelial cells. Exp Hematol 23: 41-48, 1995.

23. Pigott R, Dillon LP, Hemingway IH and Gearing AJ: Soluble forms of E-selectin, ICAM-1 and VCAM-1 are present in the supernatant of cytokine activated cultured endothelial cells. Biochem Biophys Res Commun 187: 584-589, 1992.

24. Dejana E and Giampietro C: Vascular endothelial-cadherin and vascular stability. Curr Opin Hematol 19: 218-223, 2012.

25. Le Guelte A and Gavard J: Role of endothelial cell-cell junctions in endothelial permeability. Methods Mol Biol 763: 265-279, 2011.

26. Kim S, Bakre M, Yin H and Varner JA: Inhibition of endothelial cell survival and angiogenesis by protein kinase A. J Clin Invest 110: 933-941, 2002.

27. Widlak P: The DFF40/CAD endonuclease and its role in apoptosis. Acta Biochim Pol 47: 1037-1044, 2000.

28. Liebermann DA and Hoffman B: MyD genes in negative growth control. Oncogene 17: 3319-3329, 1998.

29. Shakibaei M, Schulze-Tanzil G, Takada Y and Aggarwal BB: Redox regulation of apoptosis by members of the TNF superfamily. Antioxid Redox Signal 7: 482-496, 2005.

30. Kohler EE, Cowan CE, Wary KK, et al: NANOG induction of fetal liver kinase-1 (FLK1) transcription regulates endothelial cell proliferation and angiogenesis. Blood 117: 1761-1769, 2011.

31. Zhou W, Lin L, Evans SM, et al: Modulation of morphogenesis by noncanonical Wnt signaling requires ATF/CREB familymediated transcriptional activation of TGF $\beta 2$. Nat Genet 39: 1225-1234, 2007.
32. Nakajima M, Ogawa M, Shimoda Y, et al: Presenilin-1 controls the growth and differentiation of endothelial progenitor cells through its beta-catenin-binding region. Cell Biol Int 30: 239-243, 2006.

33. Mustonen T and Alitalo K: Endothelial receptor tyrosine kinases involved in angiogenesis. J Cell Biol 129: 895-898, 1995.

34. Zhou $\mathrm{C}$ and Wu S: T-type calcium channels in pulmonary vascular endothelium. Microcirculation 13: 645-656, 2006.

35. van der Velden AW and Thoma AA: The role of the 5 untranslated region of an mRNA in translation regulation during development. Int J Biochem Cell Biol 31: 87-106, 1999.

36. Broxmeyer HE and Williams DE: The production of myeloid blood cells and their regulation during health and disease. Crit Rev Oncol Hematol 8: 173-226, 1988.

37. Zumkeller W: The insulin-like growth factor system in hematopoietic cells. Leuk Lymphoma 43: 487-491, 2002.

38. Doh KO, Jung HK, Moon IJ, Kang HG, Park JH and Park JG: Prevention of CCl4-induced liver cirrhosis by ribbon antisense to transforming growth factor- $\beta 1$. Int J Mol Med 21: 33-39, 2008.

39. Skobin V, Jelkmann W, Morschakova E, et al: Tumor necrosis factor-alpha and TNF-beta inhibit clonogenicity of mobilized human hematopoietic progenitors. J Interferon Cytokine Res 20: $507-510,2000$

40. Broxmeyer HE, Cooper S, Li ZH, et al: Macrophage-stimulating protein, a ligand for the RON receptor protein tyrosine kinase, suppresses myeloid progenitor cell proliferation and synergizes with vascular endothelial cell growth factor and members of the chemokine family. Ann Hematol 73: 1-9, 1996.

41. Cheng LM and Wang QR: Hematopoietic inhibitors elaborated by bone marrow endothelial cells. Zhongguo Shi Yan Xue Ye Xue Za Zhi 10: 485-491, 2002 (In Chinese). 